\title{
T-INTERVAL-VALUED FUZZY SUBGROUPS AND RINGS
}

\author{
HeE WON KANG*
}

\begin{abstract}
We introduce the concepts of interval-valued fuzzy subgroups [resp. normal subgroups, rings and ideals] and investigate some of it's properties.
\end{abstract}

\section{Introduction}

In 1975, Zadeh[8] suggested the notion of interval-valued fuzzy sets as another generalization of fuzzy sets. After that time, Biswas[1] applied it to group theory, and also Kang and Hur[4] applied it to group and ring theory. Gorzalczany[2] suggested a method of inference in approximate reasoning by using interval-valued fuzzy sets. Moreover Montal and Samanta[6] introduced the concept of topology of interval-valued fuzzy sets and investigate some of it's properties. Recently, Hur et. al[3] studies interval-valued fuzzy relations in the sense of a lattice theory. In this paper, we introduce the concept of t-interval-valued fuzzy subgroups [resp.normal subgroup, rings and ideals] and investigate some of it's properties.

\section{Preliminaries}

In this section, we list some concepts and results related to intervalvalued fuzzy set theory and needed in next sections.

Let $D(I)$ be the set of all closed subintervals of the unit interval $I=[0,1]$. The elements of $D(I)$ are generally denoted by capital letters

Received September 14, 2010. Accepted October 23, 2010.

2000 Mathematics Subject Classification : 20N25.

Keywords and phrases. t-norm: t-interval-valued fuzzy subgroup[ring and ideal], t-interval-valued fuzzy normal subgroup.

* This paper was supported by Woosuk University in 2010. 
$M, N, \cdots$, and note that $M=\left[M^{L}, M^{U}\right]$, where $M^{L}$ and $M^{U}$ are the lower and the upper end points respectively. Especially, we denoted , $\mathbf{0}$ $=[0,0], \mathbf{1}=[1,1]$, and $\boldsymbol{a}=[a, a]$ for every $\boldsymbol{a} \in(0,1)$, We also note that

(i) $(\forall M, N \in D(I))\left(M=N \Leftrightarrow M^{L}=N^{L}, M^{U}=N^{U}\right)$,

(ii) $(\forall M, N \in D(I))\left(M \leq N \Leftrightarrow M^{L} \leq N^{L}, M^{U} \leq N^{U}\right)$.

For every $M \in D(I)$, the complement of $M$, denoted by $M^{C}$, is defined by $M^{C}=1-M=\left[1-M^{U}, 1-M^{L}\right](\operatorname{See}[6])$.

Definition 2.1[6,8]. A mapping $A: X \rightarrow D(I)$ is called an intervalvalued fuzzy set(is short, IVFS) in $X$, denoted by $A=\left[A^{L}, A^{U}\right]$, if $A^{L}, A^{L} \in I^{X}$ such that $A^{L} \leq A^{U}$, i.e., $A^{L}(x) \leq A^{U}(x)$ for each $x \in X$, where $A^{L}(x)\left[\operatorname{resp} A^{U}(x)\right]$ is called the lower[resp upper] end point of $x$ to $A$. For any $[a, b] \in D(I)$, the interval-valued fuzzy $A$ in $X$ defined by $A(x)=\left[A^{L}(x), A^{U}(x)\right]=[a, b]$ for each $x \in X$ is denoted by $\widetilde{[a, b]}$ and if $a=b$, then the IVFS $[\widetilde{a, b}]$ is denoted by simply $\widetilde{a}$. In particular, $\widetilde{0}$ and $\widetilde{1}$ denote the interval-valued fuzzy empty set and the interval-valued fuzzy whole set in $X$, respectively.

We will denote the set of all IVFSs in $X$ as $D(I)^{X}$.It is clear that $\mathrm{A}=[A, A] \in D(I)^{X}$ for each $A \in I^{X}$.

Definition 2.2[6]. An IVFS A is called an interval-valued fuzzy point(in short, IVFP) in $X$ with the support $x \in X$ and the value $[a, b] \in D(I)$ with $b>0$, denoted by $A=x_{[a, b]}$, if for each $y \in X$

$$
A(y)=\left\{\begin{array}{c}
{[a, b] \text { if } y=x} \\
0 \text { otherwise }
\end{array}\right.
$$

In particular, if $b=a$, then $x_{[a, b]}$ is denoted by $x_{a}$.

We will denote the set of all IVFPs in $X$ as $\operatorname{IVF}_{\mathrm{P}}(\mathrm{X})$. 
Definition $2.3[6]$. Let $A, B \in D(I)^{X}$ and let $\left\{A_{\alpha}\right\}_{\alpha \in \Gamma} \subset D(I)^{X}$. Then:

(i) $A \subset B$ iff $A^{L} \leq B^{L}$ and $A^{U} \leq B^{U}$.

(ii) $A=B$ iff $A \subset B$ and $B \subset A$.

(iii) $A^{C}=\left[1-A^{U}, 1-A^{L}\right]$.

(iv) $A \cup B=\left[A^{L} \vee B^{L}, A^{U} \vee B^{U}\right]$.

(iv) $\bigcup_{\alpha \in \Gamma} A_{\alpha}=\left[\bigvee_{\alpha \in \Gamma} A_{\alpha}^{L}, \bigvee_{\alpha \in \Gamma} A_{\alpha}^{U}\right]$

(v) $A \cap B=\left[A^{L} \wedge B^{L}, A^{U} \wedge B^{U}\right]$.

$(\mathrm{v})^{\prime} \bigcap_{\alpha \in \Gamma} A_{\alpha}=\left[\bigwedge_{\alpha \in \Gamma} A_{\alpha}^{L}, \bigwedge_{\alpha \in \Gamma} A_{\alpha}^{U}\right]$

Result 2.B $\left[6\right.$, Theorem 1]. Let $A, B, C \in D(I)^{X}$ and let $\left\{A_{\alpha}\right\}_{\alpha \in \Gamma} \subset$ $D(I)^{X}$. Then:
(a) $\widetilde{0} \subset \mathrm{A} \subset \widetilde{1}$.
(b) $A \cup B=B \cup A, A \cap B=B \cap A$.
(c) $A \cup(B \cup C)=(A \cup B) \cup C, A \cap(B \cap C)=(A \cap B) \cap C$.
(d) $A, B \subset A \cup B, A \cap B \subset A, B$.
(e) $A \cap\left(\bigcup_{\alpha \in \Gamma} A_{\alpha}\right)=\bigcup_{\alpha \in \Gamma}\left(A \cap A_{\alpha}\right)$.
(f) $A \cup\left(\bigcap_{\alpha \in \Gamma} A_{\alpha}\right)=\bigcap_{\alpha \in \Gamma}\left(A \cup A_{\alpha}\right)$.
(g) $(\widetilde{0})^{c}=\widetilde{1},(\widetilde{1})^{c}=\widetilde{0}$.
(h) $\left(A^{c}\right)^{c}=A$.
(i) $\left(\bigcup_{\alpha \in \Gamma} A_{\alpha}\right)^{c}=\bigcap_{\alpha \in \Gamma} A_{\alpha}^{c},\left(\bigcap_{\alpha \in \Gamma} A_{\alpha}\right)^{c}=\bigcup_{\alpha \in \Gamma} A_{\alpha}^{c}$.

Definition 2.4[6]. Let $A \in D(I)^{X}$ and let $x_{M} \in \operatorname{IVF}_{\mathrm{P}}(\mathrm{X})$. Then $x_{M}$ is said to belong to $A$, denoted by $x_{M} \in A$, if $M^{L} \leq A^{L}(x)$ and $M^{U} \leq A^{U}(x)$ for each $x \in X$.

It is obvious that $A=\bigcup_{x_{M} \in A} x_{M}$ and $x_{M} \in A$ if and only if $x_{M^{L}} \in A^{L}$ and $x_{M^{U}} \in A^{U}$.

Definition 2.5[7]. A t-norm is a mapping $t: I \times I \rightarrow I$ satisfing the following conditions : for any $x, y, z, u, v \in I$
(i) $t(x, y)=t(y, x)$, i.e., $x t y=y t x$.
(ii) $x t(y t z)=(x t y) t z$.
(iii) If $x \leq u$ and $y \leq v$, then $x t y \leq u t v$ 
In particular, if $y \leq v$, then $x t y \leq x t v$.

(iv) $x t 1=x$ and $x t 0=0$.

t-norms which are frequently encountered are :

(a) $x t_{0} y=\min \{x, y\}$ for $x, y \in I$.

(b) $x t_{1} y=\operatorname{Prod}\{x, y\}=x y$ for $x, y \in I$.

(c) $x t_{2} y=\max \{x+y-1,0\}$ for $x, y \in I$.

Definition 2.6[7]. A t-conorm or $s$-norm is a mapping $s_{t}: I \rightarrow I$ defined by : for any $u, v \in I$,

$$
u s_{t} v=1-(1-u) t(1-v) \text {. }
$$

It is clear that $s_{t}$ satisfies the following conditions : for any $x, y, z, u, v \in$ $I$.

(i) $x s_{t} y=y s_{t} x$.

(ii) $x s_{t}\left(y s_{t} z\right)=\left(x s_{t} y\right) s_{t} z$.

(iii) If $x \leq u$ and $y \leq v$, then $x s_{t} y \leq u s_{t} v$

In particular, if $y \leq v$, then $x s_{t} y \leq x s_{t} v$.

(iv) $x s_{t} 0=x$ and $x s_{t} 1=1$.

t-conorms corresponding to the above t-norms $t_{0}, t_{1}, t_{2}$ are as follows:

$\left(\mathrm{a}^{\prime}\right) x s_{0} y=\max \{x, y\}$ for any $x, y \in I$.

$\left(\mathrm{b}^{\prime}\right) x s_{1} y=x+y-x y$ for any $x, y \in I$.

$\left(c^{\prime}\right) x s_{2} y=\min \{1, x+y\}$ for any $x, y \in I$.

\section{3. t-interval-valued fuzzy subgroupoids}

Definition 3.1. Let $(G, \cdot)$ be a groupoid and let $A, B \in D(I)^{G}$. Then the interval-valued fuzzy product of $A$ and $B$ under $t$-norm $t$ (in short, $t$-interval-valued fuzzy product of $A$ and $B$ ), denoted by $A \circ_{t} B$, is an IVFS in $G$ defined as follows : For each $x \in G$,

$$
\left(A \circ_{t} B\right)(x)= \begin{cases}{\left[\bigvee_{y z=x}\left[A^{L}(y) t B^{L}(z)\right], \bigvee_{y z=x}\left[A^{U}(y) t B^{U}(z)\right]\right]} & \text { if } y z=x \\ 0 & \text { otherwise. }\end{cases}
$$

It is clear that $\left(D(I)^{G}, o_{t}\right)$ is a groupoid. 
Proposition 3.2. Let " $\circ$ " be same as above, let $x_{M}, y_{N} \in \operatorname{IVFp}(G)$ and let $A, B \in D(I)^{G}$. Then:
(a) $x_{M} \circ_{t} y_{N}=(x y)_{\left[M^{L} t N^{L}, M^{U} t N^{U}\right]}$.
(a) $A \circ_{t} B=\bigcup_{x_{M} \in A, y_{N} \in B} x_{M} \circ_{t} y_{N}$.

Proof. (a) Let $z \in G$. Then

$$
\begin{aligned}
\left(x_{M} \circ_{t} y_{N}\right)(z)= & \left\{\begin{array}{lr}
{\left[\bigvee_{z=x^{\prime} y^{\prime}}\left(x_{M^{L}}\left(x^{\prime}\right) \wedge y_{N^{L}}\left(y^{\prime}\right)\right), \bigvee_{z=x^{\prime} y^{\prime}}\left(x_{M^{U}}\left(x^{\prime}\right) \wedge y_{N^{U}}\left(y^{\prime}\right)\right)\right]} \\
0 & \text { if } x^{\prime} y^{\prime}=z, \\
\text { otherwise. }
\end{array}\right. \\
& = \begin{cases}{\left[M^{L} t N^{L}, M^{U} t N^{U}\right]} & \text { if } z=x y \\
0 & \text { otherwise. }\end{cases} \\
& =(x y)_{\left[M^{L} t N^{L}, M^{U} t N^{U}\right]}
\end{aligned}
$$

(b) Let $C=\bigcup_{x_{M} \in A, y_{N} \in B} x_{M} \circ_{t} y_{N}$, i.e.,

$$
C=\left[\bigvee_{x_{M^{L}} \in A^{L}, y_{N} \in^{L}}\left(x_{M^{L}} \circ_{t} y_{N^{L}}\right), \bigvee_{x_{M^{U}} \in A^{U}, y_{N^{U}} \in B^{U}}\left(x_{M^{U}} \circ_{t} y_{N^{U}}\right)\right] .
$$

For each $z \in G$, we may assume that $\exists u, v \in X$ such that $u v=z$, $x_{M}(u) \neq \mathbf{0}$ and $y_{N}(v) \neq \mathbf{0}$, i.e., $x_{M}{ }^{L}(u)>0, x_{M}{ }^{U}<1$ and $y_{N}{ }^{L}(v)>$ $0, y_{M}^{U}(v)<1$, whitout loss of generality. Then

$$
\begin{aligned}
& \left(A \circ_{t} B\right)^{L}(z)=\bigvee_{z=u v}\left[A^{L}(u) t B^{L}(v)\right] \\
& \geq \bigvee_{z=u v}\left(\bigvee_{x_{M}{ }^{L} \in A^{L}, y_{N} \in_{B^{L}}}\left[x_{M^{L}}(u) t y_{N^{L}}(v)\right]\right) \text { [Since } t \text { is increasing] } \\
& =\left(\bigcup_{x_{M^{L}} \in A^{L}, y_{N^{L}} \in B^{L}} x_{M^{L} \circ_{t}} y_{N^{L}}\right) \\
& =C^{L}(z) .
\end{aligned}
$$


Since $u_{A(u)} \in A$ and $v_{B(v)} \in B$,

$$
\begin{aligned}
& C^{L}(z)=\bigvee_{x_{M^{L}} \in A^{L}, y_{N} L \in B^{L}}\left(\bigvee_{z=u v}\left[x_{M^{L}}(u) t y_{N^{L}}(v)\right]\right) \\
& =\bigvee_{z=u v}\left(\bigvee_{x_{M^{L}} \in A^{L}, y_{N} \in^{L}}\left[x_{M^{L}}(u) t y_{N^{L}}(v)\right]\right) \\
& \geq \bigvee_{z=u v}\left[u_{A^{L}(u)}(u) t v_{B^{L}(v)}(v)\right] \\
& =\bigvee_{z=u v}\left[A^{L}(u) t B^{L}(v)\right] \\
& =\left(A \circ_{t} B\right)^{L}(z) .
\end{aligned}
$$

Thus $\left(A \circ_{t} B\right)^{L}=C^{L}$. By the similar arguments, we have $\left(A \circ_{t} B\right)^{U}=$ $C^{U}$.

Hence

$$
A \circ_{t} B=\bigcup_{x_{M^{L}} \in A^{L}, y_{N} L \in B^{L}} x_{M^{L}} \circ_{t} y_{N^{L}}
$$

Remark 3.2. Proposition 3.2 is the generalization of Proposition 3.2 in [4].

The following is the immediate result of Definition 3.1.

Proposition 3.3. Let $(G, \cdot)$ be a groupoid, and let " $\circ$ " be same as above. $D(I)^{G}$.

(a) if "." is associative[resp. commutative] in $G$, then so is " $\circ_{t}$ " in

(b) if "." is has an identity $e \in G$, then $e_{\mathbf{1}} \in \operatorname{IVFp}(G)$ is an identity of " $\circ_{t}$ " in $D(I)^{G}$, i.e., $A \circ_{t} e_{\mathbf{1}}=A=e_{\mathbf{1}} \circ_{t} A$ for each $A \in D(I)^{G}$.

Definition 3.4. Let $(G, \cdot)$ be a groupoid and let $\widetilde{0} \neq A \in D(I)^{G}$. Then $A$ is called an interval-valued fuzzy subgroupoid (in short, $t-I V G P$ ) in $G$ if $A \circ_{t} A \subset A$, i.e., $A^{L} \circ_{t} A^{L} \subset A^{L}$ and $A^{U} \circ_{t} A^{U} \subset A^{U}$.

It is clear that $\mathbf{0}$ and $\mathbf{1}$ are both $t-I V G P s$ in $G$.

The followings are the immediate results of Definitions 3.1 and 3.4. 
Proposition 3.5. Let $(G, \cdot)$ be a groupoid and let $\widetilde{0} \neq A \in D(I)^{G}$. Then the followings are equivalent:

(a) $A$ is a $t$-IVGP in $G$.

(b) For any $x_{M}, y_{N} \in A, x_{M} \circ_{t} y_{N} \in A$, i.e., $\left(A, \circ_{t}\right)$ is a groupoid.

(c) For any $x, y \in G, A^{L}(x y) \geq A^{L}(x) t A^{L}(y)$ and $A^{U}(x y) \geq$ $A^{U}(x) t A^{U}(y)$.

Remark 3.5. Proposition 3.5 is the generalization of Proposition 3.5 in [4].

Proposition 3.6. Let $A$ be a $t$-IVGP in a groupoid $(G, \cdot)$.

(a) If "." is associative in $G$, then so is " $\circ_{t}$ " in $A$, i.e., for any $x_{L}, y_{M}, z_{N} \in A$,

$x_{L} \circ_{t}\left(y_{M} \circ_{t} z_{N}\right)=\left(x_{L} \circ_{t} y_{M}\right) \circ_{t} z_{N}$.

(b) If "." is commutative in $G$, then so is "० $t$ " in $A$, i.e., for any $x_{L}, y_{M} \in A$,

$x_{L} \circ_{t} y_{M}=y_{M} \circ_{t} x_{L}$.

(c) If "." has an identity $e \in G$, then

$e_{1} \circ_{t} x_{L}=x_{L}=x_{L} \circ_{t} e_{1}, \forall x_{L} \in A$.

Remark 3.6. Proposition 3.6 is the generalization of Proposition 3.6 in [4].

From Proposition 3.5, we can define a t-IVGP in $G$ as follows.

Definition 3.4' An interval-valued fuzzy set $A$ in $G$ is called a $t$ interval-valued fuzzy subgroupoid(in short, $t$-IVGP) in $G$ if

$A^{L}(x y) \geq A^{L}(x) t A^{L}(y)$ and $A^{U}(x y) \geq A^{U}(x) t A^{U}(y), \forall x, y \in G$.

The following is the immediate result of Definition $3.4^{\prime}$.

Proposition 3.7. Let $T$ be a subset of a groupoid $(G, \cdot)$. Then $A=$ $\left[\chi_{T}, \chi_{T}\right]$ is a $t$-IVGP in $G$ if and only if $\mathrm{T}$ is a subgroupoid of $G$, where $\chi_{T}$ is the charecteristic function of $T$.

Remark 3.7. Proposition3.7 is the generalization of Proposition 3.7 in [4]. 
Definition 3.8[7]. A $t$ - norm $t$ is said to be continuous if $t: I \times I \rightarrow I$ is continuous with respect to the usual topologies.

It is clear that $t_{0}, t_{1}$ and $t_{2}$ are all continuous $t$-norms.

Proposition 3.9. Let $\left\{A_{\alpha}\right\}_{\alpha \in \Gamma}$ be any family of $t$-IVGPs in a groupoid $(G, \cdot)$. If $t$ is continuous, then $\bigcap_{\alpha \in \Gamma} A_{\alpha}$ is a $t$-IVGP in $G$.

Proof. Let $A=\bigcap_{\alpha \in \Gamma} A_{\alpha}$ and let $x, y \in G$. Then

$$
\begin{aligned}
& A^{L}(x y)=\bigwedge_{\alpha \in \Gamma} A_{\alpha}^{L}(x y) \\
& \geq \bigwedge_{\alpha \in \Gamma}\left[A_{\alpha}^{L}(x) t A_{\alpha}^{L}(y)\right] .\left[\text { Since } A_{\alpha} \text { is a } t \text {-IVGP in } G\right]
\end{aligned}
$$

Since $t$ is continous, $t$ is continuous at $\left(\bigwedge_{\alpha \in \Gamma} A_{\alpha}{ }^{L}(x), \bigwedge_{\alpha \in \Gamma} A_{\alpha}{ }^{L}(y)\right)$. Let $\epsilon>0$. Then there exists a $\delta>0$ such that if $r_{1} \geq \bigwedge_{\alpha \in \Gamma} A_{\alpha}{ }^{L}(x)+\delta$ and $r_{2} \geq \bigwedge_{\alpha \in \Gamma} A_{\alpha}{ }^{L}(y)+\delta$, then $r_{1} t r_{2} \geq\left(\bigwedge_{\alpha \in \Gamma} A_{\alpha}{ }^{L}(x)\right) t\left(\bigwedge_{\alpha \in \Gamma} A_{\alpha}{ }^{L}(y)\right)+$ $\epsilon$. Let us choose $\alpha_{0} \in \Gamma$ such that $A_{\alpha_{0}}{ }^{L}(x) \geq \bigwedge_{\alpha \in \Gamma} A_{\alpha_{0}}{ }^{L}(x)+\delta$ and $A_{\alpha_{0}}{ }^{L}(y) \geq \bigwedge_{\alpha \in \Gamma} A_{\alpha}{ }^{L}(y)+\delta$. Then

$$
A_{\alpha_{0}}{ }^{L}(x) t A_{\alpha_{0}}{ }^{L}(y) \geq\left(\bigwedge_{\alpha \in \Gamma} A_{\alpha}{ }^{L}(x)\right) t\left(\bigvee_{\alpha \in \Gamma} A_{\alpha}{ }^{L}(y)\right)+\epsilon .
$$

Thus

$$
\bigwedge_{\alpha \in \Gamma}\left[A_{\alpha_{0}}{ }^{L}(x) t A_{\alpha_{0}}{ }^{L}(y)\right] \geq\left(\bigwedge_{\alpha \in \Gamma} A_{\alpha}{ }^{L}(x)\right) t\left(\bigvee_{\alpha \in \Gamma} A_{\alpha}{ }^{L}(y)\right)
$$

So

$$
\bigwedge_{\alpha \in \Gamma}\left[A_{\alpha}^{L}(x) \wedge A_{\alpha}^{L}(y)\right] \geq\left(\bigcap_{\alpha \in \Gamma} A_{\alpha}^{L}\right)(x) t\left(\bigcap_{\alpha \in \Gamma} A_{\alpha}^{L}\right)(y)=A^{L}(x) t A^{L}(y) .
$$

Similarly, we can see that $A^{U}(x y) \geq A^{U}(x) t A^{U}(y)$. Hence $\bigcap_{\alpha \in \Gamma} A_{\alpha}$ is a $t$-IVGP in $G$.

Remark 3.9. Since $t_{0}=" \wedge "$ is continuous, Proposition 3.9 is the generalization of Proposition 3.8 in [4].

\section{4. t-interval-valued fuzzy subgroups}


Definition 4.1. Let be a group and let $A \in D(I)^{G}$. Then $A$ is called an interval-valued fuzzy subgroup under a $t$-norm $t$ (in short, $t$ - $I V G$ ) in $G$ if it satisfies the conditions : For any $x, y \in G$,

(i) $A^{L}(x y) \geq A^{L}(x) t A^{L}(y)$ and $A^{U}(x y) \leq A^{U}(x) t A^{U}(y)$,

(ii) $A^{L}\left(x^{-1}\right) \geq A^{L}(x)$ and $A^{U}\left(x^{-1}\right) \geq A^{U}(x)$.

Proposition 4.2. Let $A$ be a $t$-IVG in a group $G$. Then $A\left(x^{-1}\right)=A(x)$ for each $x \in G$.

Proof. Let $x \in G$. Then

$$
A^{U}(x)=A^{U}\left(\left(x^{-1}\right)^{-1}\right) \geq A^{U}\left(x^{-1}\right) \geq A^{U}(x) .
$$$$
A^{L}(x)=A^{L}\left(\left(x^{-1}\right)^{-1}\right) \geq A^{L}\left(x^{-1}\right) \geq A^{L}(x)
$$

and

Thus $A^{L}\left(x^{-1}\right)=A^{L}(x)$ and $A^{U}\left(x^{-1}\right)=A^{U}(x)$. So $A\left(x^{-1}=A(x)\right.$ for each $x \in X$.

Proposition 4.3. If $A$ is a $t$-IVG in a group $G$, then $H=\{x \in G$ : $A(x)=\mathbf{1}\}$ is a subgroup of $G$.

Proof. Let $x, y \in H$. Then

and

$$
A^{L}\left(x y^{-1}\right) \geq A^{L}(x) t A^{L}\left(y^{-1}\right)=A^{L}(x) t A^{L}(y)=1 t 1=1
$$

$$
A^{U}\left(x y^{-1}\right) \geq A^{U}(x) t A^{U}\left(y^{-1}\right)=A^{U}(x) t A^{U}(y)=1 t 1=1 .
$$

Thus $A^{L}\left(x y^{-1}\right)=1$ and $A^{U}\left(x y^{-1}\right)=1$. So $x y^{-1} \in H$. Hence $H$ is a subgroup of $X$.

Proposition 4.4. If $A$ is a $t$-IVG in a group $G$ and if there is a sequence $x_{n}$ in $X$ such that $\lim _{n \rightarrow \infty} A^{L}\left(x_{n}\right) t A^{L}\left(x_{n}\right)=1$ and $\lim _{n \rightarrow \infty} A^{U}\left(x_{n}\right) t A^{U}$ $\left(x_{n}\right)=1$, then $A(e)=\mathbf{1}$, where $e$ is the identity in $G$.

Proof. Let $x \in G$. Then

and

$$
A^{L}(e)=A^{L}\left(x x^{-1}\right) \geq A^{L}(x) t A^{L}\left(x^{-1}\right)=A^{L}(x) t A^{L}(x)
$$

$$
A^{U}(e)=A^{U}\left(x x^{-1}\right) \geq A^{U}(x) t A^{U}\left(x^{-1}\right)=A^{U}(x) t A^{U}(x) .
$$

Then, for each $\mathrm{n}$,

and

$$
A^{L}(e) \geq A^{L}\left(x_{n}\right) t A^{L}\left(x_{n}\right)
$$

$$
A^{u}(e) \geq A^{u}\left(x_{n}\right) t A^{u}\left(x_{n}\right) .
$$

On the other hand,

$$
1 \geq A^{L}(e) \geq \lim _{n \rightarrow \infty} A^{L}\left(x_{n}\right) t A^{L}\left(x_{n}\right)=1
$$


and

$1 \geq A^{U}(e) \geq \lim _{n \rightarrow \infty} A^{U}\left(x_{n}\right) t A^{U}\left(x_{n}\right)=1$.

Hence $A(e)=\mathbf{1}$.

Proposition 4.5. Let $A$ be a $t$-IVG in a group $G$. If $A\left(x y^{-1}\right)=\mathbf{1}$, then $A(x)=A(y)$.

Proof. Let $x, y \in G$. Then

$$
\begin{aligned}
& A^{L}(x)=A^{L}\left(\left(x y^{-1}\right) y\right) \geq A^{L}\left(x y^{-1}\right) t A^{L}(y)=1 t A^{L}(y) \\
& =A^{L}(y)=A^{L}\left(y^{-1}\right)=A^{L}\left(x^{-1}\left(x y^{-1}\right)\right) \\
& \geq A^{L}\left(x^{-1}\right) t A^{L}\left(x y^{-1}\right)=A^{L}(x) t 1=A^{L}(x)
\end{aligned}
$$

and

$A^{U}(x)=A^{U}\left(\left(x y^{-1}\right) y\right) \geq A^{U}\left(x y^{-1}\right) t A^{U}(y)=1 t A^{U}(y)$

$=A^{U}(y)=A^{U}\left(y^{-1}\right)=A^{U}\left(x^{-1}\left(x y^{-1}\right)\right)$

$\geq A^{U}\left(x^{-1}\right) t A^{U}\left(x y^{-1}\right)=A^{U}(x) t 1=A^{U}(x)$.

Hence $A(x)=A(y)$.

Proposition 4.6. Let $G$ be a group and let $0 \neq A \in D(I)^{G}$ with $A(e)=\mathbf{1}$. Then $A$ is a $t$-IVG in $G$ if and only if $A^{L}\left(x y^{-1}\right) \geq A^{L}(x) t A^{L}(y)$ and $A^{U}\left(x y^{-1}\right) \geq A^{U}(x) t A^{U}(y)$ for any $x, y \in G$.

Proof. $(\Rightarrow)$ : Suppose $A$ is a $t$-IVG in $G$ and let $x, y \in G$. Then, by Proposition 4.2, $A^{L}\left(x y^{-1}\right) \geq A^{L}(x) t A^{L}(y)$ and $A^{U}\left(x y^{-1}\right) \geq A^{U}(x) t A^{U}(y)$.

$(\Leftarrow)$ : Suppose the necessary conditions hold and let $x, y \in G$. Then

$A^{L}\left(x^{-1}\right)=A^{L}\left(e x^{-1}\right) \geq A^{L}(e) t A^{L}(x)$

$=1 t A^{L}(x)=A^{L}(x)$

and

$A^{U}\left(x^{-1}\right)=A^{U}\left(e x^{-1}\right) \geq A^{U}(e) t A^{U}(x)$

$=1 t A^{U}(x)=A^{U}(x)$.

So $A^{L}\left(x^{-1}\right) \geq A^{L}(x)$ and $A^{U}\left(x^{-1}\right) \geq A^{U}(x)$ for each $x \in G$.

On the other hand,

$$
A^{L}(x y)=A^{L}\left(x\left(y^{-1}\right)^{-1}\right) \geq A^{L}(x) t A^{L}\left(y^{-1}\right)
$$

$\geq A^{L}(x) t A^{L}(y)$

and

$$
A^{U}(x y)=A^{U}\left(x\left(y^{-1}\right)^{-1}\right) \geq A^{U}(x) t A^{U}\left(y^{-1}\right)
$$

$\geq A^{U}(x) t A^{U}(y)$

Hence $A$ is a $t$-IVG in $G$.

Proposition 4.7. Let $G_{p}$ be the cyclic group of prime order $p$ and let $A \in D(I)^{G_{p}}$ with $A(e)=\mathbf{1}$, where $e$ is the identity in $G_{p}$. If $A(x)=$ 
$A(a) \leq A(e)$, for each $e \neq x \in G_{p}$ where $G_{p}=(a)=e=a^{0}, a^{1}, a^{2}, \cdots a^{p-1}$, then $A$ is a $t$-IVG in $G_{p}$.

Proof. Let $x, y \in G_{p}$.

Case(i) : Suppose $x \neq e, y \neq e$ and $x y^{-1} \neq e$. Then, by the hypothesis,

$$
A^{L}\left(x y^{-1}\right)=A^{L}(x)=A^{L}(y)
$$

and

$$
A^{U}\left(x y^{-1}\right)=A^{U}(x)=A^{U}(y) .
$$

Thus

$$
A^{L}\left(x y^{-1}\right) \geq A^{L}(x) t A^{L}(y)
$$

and

$$
A^{U}\left(x y^{-1}\right) \geq A^{U}(x) t A^{U}(y) .
$$

Case(ii) : Suppose $x \neq e, y \neq e$ and $x y^{-1}=e$. Then, by the hypothesis,

and

$$
A^{L}(x)=A^{L}(y) \leq A^{L}(e)=A^{L}\left(x y^{-1}\right)
$$

$$
A^{U}(x)=A^{U}(y) \leq A^{U}(e)=A^{U}\left(x y^{-1}\right) .
$$

Thus

$$
A^{L}\left(x y^{-1}\right) \geq A^{L}(x) t A^{L}(y)
$$

and

$$
A^{U}\left(x y^{-1}\right) \geq A^{U}(x) t A^{U}(y) .
$$

Case(iii) : Suppose $x \neq e, y=e$ and $x y^{-1} \neq e$. Then, by the hypothesis,

$$
\begin{aligned}
& A^{L}(x)=A^{L}\left(x y^{-1}\right) \leq A^{L}(e)=A^{L}(y)=1 \\
& A^{U}(x)=A^{U}\left(x y^{-1}\right) \leq A^{U}(e)=A^{U}(y)=1 .
\end{aligned}
$$

and

Thus

and

$$
A^{L}\left(x y^{-1}\right) \geq A^{L}(x) t 1=A^{L}(x) t A^{L}(y)
$$

$A^{U}\left(x y^{-1}\right) \geq A^{U}(x) t 1=A^{L}(x) t A^{U}(y)$.

Case(iv) : Suppose $x=e, y \neq e, x y^{-1} \neq e$. Then it is the same as case (iii).

In all,

$$
A^{L}\left(x y^{-1}\right) \geq A^{L}(x) t A^{L}(y)
$$

and

$$
A^{U}\left(x y^{-1}\right) \geq A^{U}(x) t A^{U}(y) .
$$

Hence $A$ is a $t$-IVG in $G_{p}$. 
Definition 4.8. Let $A$ be a $t$-IVG in a group $G$. Then $A$ is called a t-interval-valued fuzzy normal subgroup (in short, $t$ - $I V N G$ ) in $G$ if $A(x y)=A(y x)$ for any $x, y \in X$.

Proposition 4.9. Let $A$ be a $t$-IVNG in a group $G$.

(a) For each $B \in D(I)^{G}, A \circ_{t} B=B \circ_{t} A$.

(b) If $B$ is a $t$-IVG in $G$, then so is $B \circ_{t} A$.

Proof. (a) Let $z \in G$ with $z=x y$. Then

$$
\begin{aligned}
& \left(A \circ_{t} B\right)^{L}(z)=\bigvee_{x y=z} A^{L}(x) t B^{L}(y) \\
& =\bigvee_{x=z y^{-1}} A^{L}(x) t B^{L}(y) \\
& =\bigvee_{x=z y^{-1}} A^{L}\left(z y^{-1}\right) t B^{L}(y) \\
& =\bigvee_{x^{\prime}=y^{-1} z} A^{L}\left(x^{\prime}\right) t B^{L}(y)
\end{aligned}
$$

(Since $A$ is a $t$-IVNG in $G$ )

$$
=\bigvee_{y x^{\prime}=z} B^{L}(y) t A^{L}\left(x^{\prime}\right)=\left(B \circ_{t} A\right)^{L} \text {. }
$$

Similarly, $\left(A \circ_{t} B\right)^{U}(z)=\left(B \circ_{t} A\right)^{U}(z)$. So $A \circ_{t} B=B \circ_{t} A$.

(b) By Definition 3.4 and (a),

$$
\begin{aligned}
& \left(B \circ_{t} A\right) \circ_{t}\left(B \circ_{t} A\right)=B \circ_{t}\left(A \circ_{t} B\right) \circ_{t} A \\
& =B \circ_{t}\left(B \circ_{t} A\right) \circ_{t} A \\
& =\left(B \circ_{t} B\right) \circ_{t}\left(A \circ_{t} A\right) \subset B \circ_{t} A .
\end{aligned}
$$

Thus $B \circ_{t} A$ is a $t$-IVGP in $G$. Now let $x \in G$ with $x^{-1}=y z$.

Then

$$
\begin{aligned}
& \left(B \circ_{t} A\right)^{L}\left(x^{-1}\right)=\bigvee_{y z=x^{-1}} B^{L}(y) t A^{L}(z) \\
& =\bigvee_{x=z^{-1} y^{-1}} B^{L}\left(\left(y^{-1}\right)^{-1}\right) t A^{L}\left(\left(z^{-1}\right)^{-1}\right) \\
& \geq \bigvee_{x=z^{-1} y^{-1}} B^{L}\left(y^{-1}\right) t A^{L}\left(z^{-1}\right) \\
& =\bigvee_{x=z^{-1} y^{-1}} A^{L}\left(z^{-1}\right) t B^{L}\left(y^{-1}\right) \\
& =\left(A \circ_{t} B\right)^{L}(x)=\left(B \circ_{t} A\right)^{L}(x) .(\text { By (a) })
\end{aligned}
$$

Similarly, we have $\left(B \circ_{t} A\right)^{U}\left(x^{-1}\right) \geq\left(B \circ_{t} A\right)^{U}(x)$.

Hence $B \circ_{t} A$ is a $t$-IVG in $G$.

\section{5. t-interval-valued fuzzy rings and ideals}

Definition 5.1. Let $(R,+, \cdot)$ be a ring, let $t$ be a $t$-interval-valued $f u z z y$ subring (in short, $t$-IVR) in $R$ if it satisfies the following conditions: $4.1)$,

(i) $A$ is a $t$-IVG in $R$ with respect to " + " (in the sense of Definition 
(ii) $A$ is a $t$-IVGP in $R$ with respect to "." (in the sense of Definition 3.4 or Definition $3.4^{\prime}$ ).

Proposition 5.2. Let $R$ be a ring and let $\widetilde{0} \neq A \in D(I)^{R}$ such that $A(0)=\mathbf{1}$ where 0 is the zero element for " + " in $R$. Then $A$ is a $t$-IVR in $R$ if and only if any $x, y \in G$.

and

$$
A^{L}(x) t A^{L}(y) \leq A^{L}(x-y) \wedge A^{L}(x y)
$$

$$
A^{U}(x) t A^{U}(y) \leq A^{U}(x-y) \wedge A^{U}(x y)
$$

Proof. $A$ is a $t$-IVR in $R$

if and only if

$$
A^{L}(x-y) \geq A^{L}(x) t A^{L}(y), A^{U}(x-y) \geq A^{U}(x) t A^{U}(y)
$$

and

(by Proposition 4.6)

$A^{L}(x y) \geq A^{L}(x) t A^{L}(y), A^{U}(x y) \geq A^{U}(x) t A^{U}(y)$ for any $x, y \in R$

(by Definition $3.4^{\prime}$ )

if and only if

and

$$
A^{L}(x) t A^{L}(y) \leq A^{L}(x-y) \wedge A^{L}(x y)
$$

$A^{U}(x) t A^{U}(y) \leq A^{U}(x-y) \wedge A^{U}(x y)$ for any $x, y \in R$.

Corollary $5.2[4$, Proposition 6.2]. Let $R$ be a ring and let $\widetilde{0} \neq$ $A \in D(I)^{R}$. Then $A$ is an IVR in $R$ if and only if $A^{L}(x) \wedge A^{L}(y) \leq$ $A^{L}(x-y) \wedge A^{L}(x y)$ and $A^{U}(x) \wedge A^{U}(y) \leq A^{U}(x-y) \wedge A^{U}(x y)$ for any $x, y \in R$.

Definition 5.3. Let $R$ be a ring and let $\widetilde{0} \neq A \in D(I)^{X}$ be a $t$-IVR in $R$. Then $A$ is called a:

(1) t-interval-valued fuzzy left ideal (in short, $t-I V L I$ ) in $X$ if $A^{L}(x y) \geq A^{L}(y)$ and $A^{U}(x y) \geq A^{U}(y)$ for any $x, y \in R$.

(2) $t$ - interval-valued fuzzy right ideal (in short, $t$-IVRI) in $R$ if $A^{L}(x y) \geq A^{L}(x)$ and $A^{U}(x y) \geq A^{U}(x)$ for any $x, y \in R$.

(3) t-interval-valued fuzzy ideal (in short, $t$-IVI) in $R$ if it is both $t$-IVLI and $t$-IVRL in $X$.

Proposition 5.4. Let $R$ be a ring and let $\widetilde{0} \neq A \in D(I)^{R}$ such that $A(0)=1$. Then $A$ is a $t$-IVI [resp.t-IVLI, $t$-IVRI] in $R$ if and only if $A^{L}(x-y) \geq A^{L}(x) t A^{L}(y), A^{U}(x-y) \geq A^{U}(x) t A^{U}(y)$ and $A^{L}(x y) \geq$ $A^{L}(x) s_{t} A^{L}(y)\left[\geq A^{L}(y), \geq A^{L}(x)\right], A^{U}(x y) \geq A^{U}(x) s_{t} A^{U}(y)\left[\geq A^{U}(y), \geq\right.$ 
$\left.A^{U}(x)\right]$ for any $x, y \in R$.

Proof. It is obvious from Proposition 4.2 and Definition 4.3.

Corollary 5.4 $[4$, Proposition 6.5]. Let $R$ be a ring and let $\widetilde{0} \neq$ $A \in D(I)^{R}$. Then $A$ is an [resp. IVLI, IVRI] in $R$ if and only if $A^{L}(x-y) \geq A^{L}(x) \wedge A^{L}(y), A^{U}(x-y) \geq A^{U}(x) \wedge A^{U}(y)$ and $A^{L}(x y) \geq$ $A^{L}(x) \wedge A^{L}(y)\left[\geq A^{L}(y), \geq A^{L}(x)\right], A^{U}(x y) \geq A^{U}(x) \wedge A^{U}(y)\left[\geq A^{U}(y),>\right.$ $\left.A^{U}(x)\right]$ for any $x, y \in R$.

Proposition 5.5. Let $R$ be a skew field and let $\widetilde{0} \neq A \in D(I)^{R}$. Then $A$ is a $t$-IVI in $R$ if and only if

(1) $A(x)=A(e)$, for each $x \in R-\{0\}$,

(2) $A^{L}(0)=A^{L}(0) s_{t} A^{L}(x) \geq A^{L}(x) t A^{L}(e)$

and

$A^{U}(0)=A^{U}(0) s_{t} A^{U}(e) \geq A^{U}(x) t A^{U}(e)$ for each $x \in X$,

(3) $A^{L}(e)=A^{L}(e) s_{t} A^{L}(e)$ and $A^{U}(e)=A^{U}(e) s_{t} A^{U}(e)$.

Proof. $(\Rightarrow)$ : Suppose $A$ is a $t$-IVI in $R$ and let $0 \neq x \in R$. Then

$A^{L}(x)=A^{L}(x e) \geq A^{L}(x) s_{t} A^{L}(e)$ [By Proposition 5.4]

$=A^{L}(e) s_{t} A^{L}(x) \geq A^{L}(e) s_{t} 0$

$=1-\left(1-A^{L}(e)\right) t(1-0)$

$=1-\left(1-A^{L}(e)\right)=A^{L}(e)=A^{L}\left(x^{-1} x\right)$

$\geq A^{L}\left(x^{-1}\right) s_{t} A^{L}(x)=A^{L}(x) s_{t} A^{L}\left(x^{-1}\right)$

$\geq A^{L}(x) s_{t} 0=A^{L}(x)$.

So $A^{L}(x)=A^{L}(e)$. Similarly, we can see that $A^{U}(x)=A^{U}(e)$ for each $x \in R-\{0\}$. Hence, the condition (1) holds.

Let $x \in R$. Then

$$
\begin{aligned}
& A^{L}(0)=A^{L}(x 0) \geq A^{L}(x) s_{t} A^{L}(0) \\
& =A^{L}(0) s_{t} A^{L}(x) \geq A^{L}(0) s_{t} 0=A^{L}(0) \\
& =A^{L}(0) s_{t} A^{L}(0)=A^{L}(e-e) s_{t} A^{L}(e-e) \\
& \geq\left[A^{L}(e) t A^{L}(e)\right] s_{t}\left[A^{L}(e) t A^{L}(e)\right] \\
& \geq\left[A^{L}(e) t A^{L}(e)\right] s_{t} 0=A^{L}(e) t A^{L}(e) .
\end{aligned}
$$

Thus $A^{L}(0)=A^{L}(0) s_{t} A^{L}(x) \geq A^{L}(x) t A^{L}(e)$. Similarly, we can see that $A^{U}(0)=A^{U}(0) s_{t} A^{U}(x) \geq A^{U}(x) t A^{U}(e)$. So the condition (2) holds.

Now let $0 \neq x \in R$. Then, by (1),

$$
\begin{aligned}
& A^{L}(e)=A^{L}(x)=A^{L}(x e) \geq A^{L}(x) s_{t} A^{L}(e) \\
& =A^{L}(e) s_{t} A^{L}(e) \geq A^{L}(e) s_{t} 0=A^{L}(e) .
\end{aligned}
$$


Thus $A^{L}(e)=A^{L}(e) s_{t} A^{L}(e)$. Similarly, we can see that $A^{U}(e)=$ $A^{U}(e) s_{t} A^{U}(e)$. So the condition (3) holds.

$(\Leftarrow)$ : Suppose the necessary condition hold and let $x \in R$. Since $A^{L}(0)=A^{L}(-0)$ and $A^{U}(0)=A^{U}(-0)$, let $x \neq 0$. Then, by (1),

$$
A^{L}(x)=A^{L}(e)=A^{L}(-x)
$$

and

$A^{U}(x)=A^{U}(e)=A^{U}(-x)$.

Thus

$A^{L}(-x)=A^{L}(x)$ and $A^{U}(-x)=A^{U}(x)$ for each $x \in X,(6.1)$

Let $x, y \in R$.

Case (i): Suppose $x+y \neq 0$ with $y \neq 0$. Then

$A^{L}(x+y)=A^{L}(x+y) t 1$

$\geq A^{L}(x+y) t A^{L}(x)$

$=A^{L}(e) t A^{L}(x)$

$=A^{L}(y) t A^{L}(x)$ (by (1))

$=A^{L}(x) t A^{L}(y)$ (by (1))

Similarly, we can see that $A^{U}(x+y)=A^{U}(x) t A^{U}(y)$.

Case(ii) : Suppose $x+y=0$ with $x=0$. Then

$A^{L}(x+y)=A^{L}(0)=A^{L}(0) t 1 \geq A^{L}(0) t A^{L}(y)$

$=A^{L}(x) t A^{L}(y)$

Also, we can see that $A^{U}(x+y)=A^{U}(x) t A^{U}(y)$.

Case(iii) : Suppose $x+y=0$ with $0 \neq x=-y$. Then

$A^{L}(x+y)=A^{L}(0) \geq A^{L}(e) t A^{L}(e)$ (by (2))

$=A^{L}(x) t A^{L}(-y)($ by $(1))$

$=A^{L}(x) t A^{L}(y)$. (by (6.1))

and

$A^{U}(x+y)=A^{U}(0) \geq A^{U}(e) t A^{U}(e)($ by $(2))$

$=A^{U}(x) s_{t} A^{U}(-y)$ (by (1))

$=A^{U}(x) s_{t} A^{U}(y)$. (by (6.1))

In all, for any $x, y \in X, A^{L}(x+y) \geq A^{L}(x) t A^{L}(y)$ and $A^{U}(x+y) \geq$ $A^{U}(x) t A^{U}(y)$. (6.2)

Now let $x, y \in R$.

Case(i) : Suppose $x y=0$ with, say, $x=0$.

Then, by (2),

$$
A^{L}(x y)=A^{L}(0)=A^{L}(0) s_{t} A^{L}(y)=A^{L}(x) t A^{L}(y)
$$


and

$A^{U}(x y)=A^{U}(0)=A^{U}(0) s_{t} A^{U}(y)=A^{U}(x) t A^{U}(y)$.

Case(ii) : Suppose $x y \neq 0$. Then, by (1) and (3),

$A^{L}(x y)=A^{L}(e)=A^{L}(e) s_{t} A^{L}(e)=A^{L}(x) s_{t} A^{L}(y)$

and

$$
A^{U}(x y)=A^{U}(e)=A^{U}(e) s_{t} A^{U}(e)=A^{U}(x) s_{t} A^{U}(y) .
$$

In all, for any $x, y \in R, A^{L}(x y) \geq A^{L}(x) s_{t} A^{L}(y)$ and $A^{U}(x y) \geq A^{U}(x) s_{t}$ $A^{U}(y)$. (6.3)

On the other hand, by (6.3),

$$
\begin{aligned}
& A^{L}(x y) \geq A^{L}(x) s_{t} A^{L}(y) \geq A^{L}(x) s_{t} 0 \\
& =A^{L}(x)=A^{L}(x) t 1 \geq A^{L}(x) t A^{L}(y)
\end{aligned}
$$

and

$$
\begin{aligned}
& A^{U}(x y) \leq A^{U}(x) t A^{U}(y) \leq A^{U}(x) t 1 \\
& =A^{U}(x)=A^{U}(x) s_{t} 0 \leq A^{U}(x) s_{t} A^{U}(y) .
\end{aligned}
$$

So $A^{L}(x y) \geq A^{L}(x) t A^{L}(y)$ and $A^{U}(x y) \leq A^{U}(x) s_{t} A^{U}(y)$ for any $x, y \in$ $R$. (6.4) Hence, by (6.1), (6.2), (6.3) and (6.4), $A$ is a $t$ - IVI in $R$.

Corollary 5.5[4, Proposition 6.7]. Let $R$ be a skew field and let $\widetilde{0} \neq A \in \operatorname{IVS}(R)$. Then $A$ is an IVI[resp.IVLI, IVRI] in $R$ if and only if $A(x)=A(e) \leq A(0)$, ie., $A^{L}(x)=A^{L}(e) \leq A^{L}(0)$ and $A^{U}(x)=$ $A^{U}(e) \leq A^{U}(0)$ for each $0 \neq x \in R$.

Proposition 5.6. Let $R$ be a commutative ring with a unity $e$. If for any $t$-IVI $A$ in $R, A(x)=A(e) \leq A(0)$, i.e., $A^{L}(x)=A^{L}(e) \leq A^{L}(0)$ and $A^{U}(x)=A^{U}(e) \leq A^{U}(0)$ for each $0 \neq x \in R$, then $R$ is a field.

Proof. Let $A$ be an ideal of $R$ such that $A \neq R$. Then clearly $A=\left[\chi_{A}, \chi_{A}\right]$ is a $t$-IFI in $R$ such that $A \neq \widetilde{1}$. Then there exists $y \in R$ such that $y \in A$. Thus $\chi_{A}(y)=0$. By the hypothesis, $\chi_{A}(x)=\chi_{A}(e) \leq$ $\chi_{A}(0)$ for each $0 \neq x \in R$. Thus $\chi_{A}(0)=1$ ie., $A=\{0\}$. Hence $X$ is a field.

Corollary 5.6 [4, Proposition 6.9]. Let $R$ be a commutative ring with a unity $e$. If for any IVI $A$ in $R, A(x)=A(e) \leq A(0)$, ie., $A^{L}(x)=$ $A^{L}(e) \leq A^{L}(0)$ and $A^{U}(x)=A^{U}(e) \leq A^{U}(0)$ for each $0 \neq x \in R$. Then $R$ is a field. 


\section{References}

[1] R. Biswas, Rosenfeld's fuzzy subgroups with interval-valued membership functions, Fuzzy set and systems 63(1995) 87-90.

[2] M.B.Gorzalczany, A method of inference in approximate reasoning based on interval-values fuzzy, sets, Fuzzy sets and Systems 21(1987) $1-17$.

[3] K.Hur, J.G.Lee and J.Y.Choi, Interval-valued fuzzy relations, J.Korean Institute of Intelligent systems 19(3)(2009) 425-432.

[4] H.W.Kang and K.Hur, Interval-valued fuzzy subgroups and rings, To be subunited.

[5] W.J.Liu, Fuzzy invaiant subgroups and fuzzy ileds, Fuzzy sets and Systems 8(1982) 133-189.

[6] T.K.mondal and S.K.Samanta, Topology of interval-valued fuzzy sets, Indian J. Pure Appl. Math. 30(1)(1999) 20-38.

[7] B.Schweizer and A.Sklar, Statisitical metric spaces, Pacific J.Math. 10(1960), 313-334.

[8] L.A.Zadeh, The concept of a linguistic variable and its application to approximate reasoning-I, Inform. sci 8(1975) 199-249.

*Dept of Mathematics Education,

Woosuk University,

Hujong-Ri, Samrae-Eup,

Wanju-Kun, cheonbuk, Korea 560-701

E-mail: khwon@woosuk.ac.kr 